\title{
Overcoming response bias using symbolic representations of number by chimpanzees (Pan troglodytes)
}

\author{
SARAH T. BOYSEN, KIMBERLY L. MUKOBI, and GARY G. BERNTSON \\ Ohio State University, Columbus, Ohio
}

\begin{abstract}
We previously reported that chimpanzees were unable to optimally select the smaller of two candy arrays in order to receive a larger reward. When Arabic numerals were substituted for the candy arrays, animals who had had prior training with numerical symbols showed an immediate and significant improvement in performance and were able to select reliably the smaller numeric representation in order to obtain a larger reward. Poor performance with candy arrays was interpreted as reflecting a response bias toward the intrinsic incentive and/or perceptual features of the larger array. In contrast, the Arabic numerals represent numerosity symbolically and appear to promote response choice on the basis of abstract processing of numerosity, with minimal interference from the inherent properties of the choice stimuli. The present study tested the hypothesis that, for mixed symbol-candy choice pairs, the requisite processing of the abstract numeral may foster a mode of numerical judgment that diminishes the interfering incentive/perceptual effects of the candy stimuli. The results were consistent with this hypothesis. Whereas performance on candy-candy arrays was significantly below chance levels, performance on numeral-candy choice pairs was significantly above chance and comparable with performance on numeral-numeral pairs.
\end{abstract}

Chimpanzees have been shown to demonstrate remarkable cognitive capabilities that are often strikingly reminiscent of human reasoning abilities and conceptual skills (see, e.g., Boysen, Berntson, Shreyer, \& Hannan, 1995; Fujita \& Matsuzawa, 1986; Gillan, Premack, \& Woodruff, 1981; Matsuzawa, 1985a, 1985b; Premack, 1986; Savage-Rumbaugh, 1986). For example, they have been shown to have impressive capabilities in numerical processing, symbolic representation of number, and even numerical reasoning that does not require the support of language, as typically defined (see, e.g., Boysen, 1997; Boysen \& Berntson, 1989, 1995; Matsuzawa, 1985b; Murofushi, 1997). Behavioral and cognitive differences nonetheless exist between chimpanzees and humans, and those differences can sometimes be more informative than similarities for the comparative understanding of cognitive function (Boysen, Berntson, Hannan, \& Cacioppo, 1996). Several years ago, we encountered a rather striking training failure with two chimpanzees (Boysen \&

Support for these studies was provided by NIMH Grant MH 49834 to S.T.B. The Ohio State University Chimpanzee Center is fully accredited by the American Association for the Accreditation of Laboratory Animal Care. The contributions and commitment of Nancy Brown, Valerie Kuhlmeier, Tina Gilbert, David Brown, Karen Hallberg, and Tyler Himes toward the care and training of the chimpanzees are gratefully acknowledged, with special thanks to Diane McClure and Elysse Orchard. Correspondence concerning this article should be addressed to S. T. Boysen, Department of Psychology, Rm. 48, Townshend Hall, 1885 Neil Avenue, Ohio State University, Columbus, OH 43210-1222 (e-mail: boysen.1@osu.edu).
Berntson, 1995). The task required the seemingly simple choice between two different-sized candy arrays. A reversed reinforcement contingency was imposed, so that the candies in the selected array were removed and the animal received the remaining, nonchosen array as a reward. In order to maximize payoff, it was in the best interest of the chimpanzee subject to choose the dish with the smaller number of candies, in order to reap the larger remainder. Both of the animals in the preliminary study failed to do so, and even after hundreds of training trials, they persisted in selecting the larger candy array and, thus, consistently received the smaller amount of reward.

To further explore their seeming inability to acquire more optimal performance under a relatively simple task contingency, we capitalized on the previously acquired skills of one of the animals (Sheba) in counting and in the use of Arabic numerals as representations for quantities (Boysen, 1993; Boysen \& Berntson, 1989). All the task rules and features were identical, except that Arabic numerals were substituted for the candy arrays. The same contingency applied, and the subject was rewarded with the number of candies represented by the nonselected numerical stimulus. Without further training, Sheba immediately invoked the optimal selection rule. As long as Arabic numerals served as stimuli, she consistently selected the smaller numeral and earned the greater number of candies. When candies were reintroduced as stimuli, performance dramatically fell but immediately returned to more optimal levels when Arabic numerals were again substituted for the candy stimuli. 
On the basis of these data, we suggested that there might be two incompatible response dispositions operative in this task. An associative disposition to select the smaller of the two arrays would be expected to develop on the basis of the instrumental reward contingency. The fact that such a disposition had developed was apparent when Arabic numerals were presented, resulting in more optimal performance despite the fact that this implicit knowledge did not manifest when candy arrays served as stimuli. We hypothesized that the poor performance with candy arrays was attributable to an interfering nonassociative disposition toward the direct perceptual and/or incentive features of the larger array. This may have been fostered by numerosity judgments based on subitizing or magnitude estimation-processes that depend on the immediate perceptual features of the task stimuli (see, e.g., Gallistel, 1993). We further speculated that performance with the numerals was more optimal because these stimuli promote an abstract enumeration process that is not as closely tied to the direct perceptual/incentive features of the task stimuli (Berntson, Boysen, \& Cacioppo, 1993; Boysen, 1997; Cacioppo \& Berntson, 1994). By promoting an abstract mode of processing, symbolic stimuli may direct attention to relevant aspects of their referents (numerosity), while minimizing the impact of other aspects (incentive or perceptual features).

What remains unsettled is the extent to which a symbolic representation, as an essential element in a cognitive task, might foster an alternative mode of cognitive processing of other nonsymbolic (candy array) elements. To explore this issue, in the present study we examined the performance of chimpanzees on the interference task as described above, with an added condition in which one of the choice stimuli was a candy array and the other was an Arabic numeral. We hypothesized that the need to process the numerical value of the symbol abstractly, in a less perceptually based mode, might foster a similar mode of processing of the paired candy array and, thereby, minimize the perceptual or incentive-based interference typically associated with such arrays. An alternative hypothesis predicted just the opposite -that the incentive features of the candy array, when compared with the lack of inherent incentive features of the Arabic numerals, would lead to an even more suboptimal performance. A secondary goal of the study was to evaluate the stability of the task interference effect over the approximate 6-year period since the original study.

\section{METHOD}

\section{Subjects}

Five adult chimpanzees (Pan troglodytes) served as subjects. These included 2 females (ages, 16 and 39 years) and 3 males (ages, 10,18 , and 18.5 years). All the subjects had extensive experience and training on a variety of cognitive and behavioral tasks (see, e.g., Boysen, Berntson, Shreyer, \& Quigley, 1993; Limongelli, Boysen, \& Visalberghi, 1993; Thompson, Oden, \& Boysen, 1997), as well as training with counting and symbolic representations (Arabic nu- merals) of number (Boysen, 1993, 1997; Boysen \& Berntson, 1989). Each of the animals had also participated in a previous study of the task interference effects in the present paradigm (Boysen \& Berntson, 1995; Boysen et al., 1996).

\section{Procedure}

All the subjects were tested individually in an indoor housing area. The testing room was equipped with a large polycarbonate window and a steel shelf outside the window, where the stimulus items were displayed. The chimpanzees made their choices by pointing to items, with the experimenter positioned out of view of the animal but able to clearly see their selections. All the subjects were free to exit the testing situation at any time but were readily compliant and fully participated in all phases of testing, without incident.

The task was the same interference paradigm as that employed in prior studies (Boysen \& Berntson, 1995; Boysen et al., 1996), in which the animal had to select the numerically smaller of two choice stimuli in order to receive the larger, nonselected quantity as a reward. On each trial, two arrays of candy (peanut M\&Ms or gummy bears), two Arabic numeral symbols (black numerals on a white $7.5 \times 12 \mathrm{~cm}$ background), or a combination of a candy array and an Arabic numeral were placed in two separate dishes. The two dishes were placed on the shelf in front of the subject, approximately $30 \mathrm{~cm}$ apart, and the chimpanzee was permitted to select one of the choice stimuli (by pointing). A reversed reward contingency was in effect so that the chimpanzee was reinforced with the nonselected candy array or, if an Arabic numeral was the nonselected item, a number of candies corresponding to the numeral. Once a choice was made, the experimenter removed the contents of the selected dish, and the subject received the contents of the nonselected dish, or the number of candies corresponding to the nonselected Arabic numeral. Thus, it was to the subject's advantage to select the dish containing the numerically smaller stimulus, in order to receive the larger number of candy reinforcers that remained.

Each animal was tested for a total of six sessions run on separate days: two with candy arrays, two with Arabic numerals, and two with a candy array and an Arabic numeral as choice stimuli. Numeric stimuli and array sizes ranged from zero to six, with a blockrandomized subset of choice pairs selected to sample a range of array disparities and disparity ratios (see below). Each animal received a total of 112 trials distributed over the six sessions. Candycandy and number-number sessions were made up of 18 trials, with right-left counterbalancing of the positions of the larger and the smaller arrays over trials. Candy-number sessions were similar, but 20 trials were given to accommodate the added requirement for counterbalancing the positions of the candy array and the numeral (each array-numeral combination was presented twice, with the candy positioned on the left for one trial and on the right for the other). Trials were self-paced by the subject, with the intertrial interval generally falling between 45 and $60 \mathrm{sec}$.

\section{Data Analyses}

Because of the modest $N$ and to avoid distribution assumptions, primary analyses were based on nonparametric tests. Friedman's test was used for comparisons across multiple conditions, and standard chi-square tests (with Yates correction) were used for pairwise comparisons and for tests against a fixed, chance level. In view of the use of nonparametric analyses, summary statistics are given as medians and interquartile ranges.

In addition to binomial tallies of correct responses and errors, a disparity ratio index was derived, as described in Boysen et al. (1996), because our previous studies indicated that error rates were related to both the numerical disparity among the choice stimuli and the quantity of the arrays. This ratio is the absolute value of the numerical difference between the two choice stimuli divided by the mean numerical size of the stimulus pair. This index provides a useful Weber-like summary statistic that expresses the absolute nu- 
merical disparity between the two stimuli of a choice pair, scaled by the overall numerical size of the stimulus elements. Our previous study found that the disparity ratio was more closely associated with performance than was either the absolute disparity between the stimulus pairs or the array size alone. The relation between the disparity ratio and performance was derived by individual (animal by animal) linear regressions to define the slope of the disparity ratio-performance function (see Figure 2). For purposes of analyses, these slope values were treated in a manner similar to other performance measures and were evaluated by nonparametric tests.

\section{RESULTS}

\section{Overall Analysis}

Homogeneous arrays. In accord with our previous findings, none of the 5 animals was able to reliably select the smaller of two candy arrays, despite the fact that this selection would yield the larger reward. Performance with numeral-numeral pairs or array-numeral combinations was more nearly optimal (see Figure 1). A Friedman's analysis revealed significant differences in performance between the stimulus conditions [candy-candy, numeral-numeral, and candy-numeral pairs; $\chi^{2}(d f=2)=$ $7.6, p<.03$ ]. As is illustrated in Figure 1, performance with candy-candy arrays was significantly below the expected chance performance of .50 over all the subjects [proportion correct, median $=.19 \pm .025$ interquartile range, $\left.\chi^{2}(d f=1)=62.4, p<.001\right]$ and for each individual animal [all $\chi^{2} \mathrm{~s}(d f=1)>9.0$, all $p \mathrm{~s}<.01$ ]. This confirmed our previous finding of a strong interference effect in this task when object arrays served as stimuli (Boysen \& Berntson, 1995; Boysen et al., 1996). Moreover, these findings demonstrate that the animals were

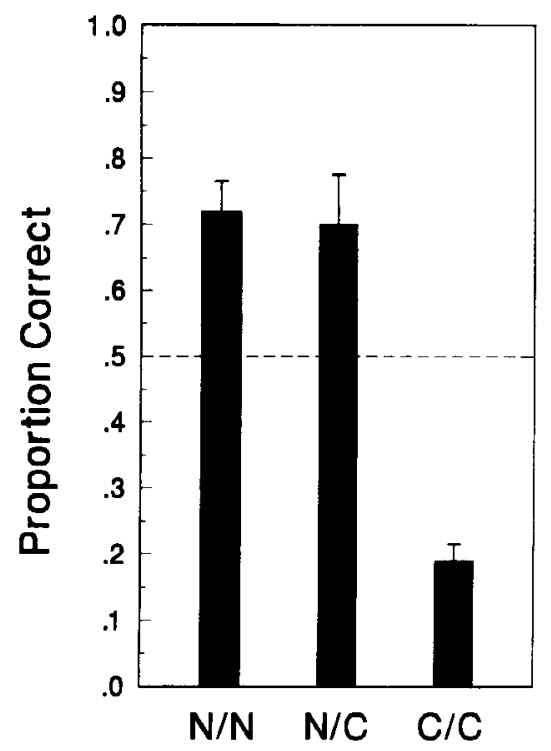

Figure 1. Overall task performance of chimpanzees as a function of choice stimuli. Data are medians and interquartile ranges. $\mathbf{N} / \mathbf{N}$, numeral-numeral choice pairs; $\mathbf{N} / \mathbf{C}$, numeral-candy array choices; $\mathrm{C} / \mathrm{C}$, candy-candy array stimuli. not insensitive to array stimuli or numerical disparities, as their performance was significantly different from chance. Rather, the animals failed to respond optimally, relative to the reward contingency. With candy-candy arrays, the animals reliably selected the larger of the two arrays, even though this choice yielded the smaller amount of candy reward.

In contrast, during numeral-numeral sessions, the performance of the animals was significantly higher than that for candy-candy sessions [proportion correct for the numeral-numeral sessions $=.72 \pm .045$ interquartile range, as compared with candy-candy sessions, $\chi^{2}$ $(d f=1)=5.0, p<.03$ ]. Moreover, as is illustrated in Figure 1, performance with numeral-numeral pairs was significantly above chance, both overall $\left[\chi^{2}(d f=1)=\right.$ $45.0, p<.001$ ] and for each individual animal [all $\chi^{2} \mathrm{~s}$ $(d f=1)>5.4$, all $p \mathrm{~s}<.02$ ]. These results are consistent with the suggestion that the intrinsic incentive/perceptual attraction to the larger candy array contributed to an inability to select the smaller array, when the choice stimuli were collections of discrete objects. Numerical symbols may effectively represent quantities without such interfering biases, thus allowing choice selection to be guided more by the reward contingencies.

Numeral-candy array pairs. The crucial test condition of the present study was the presentation of numeralcandy array pairs. We hypothesized that the Arabic numeral might promote an abstract, less perceptually based mode of processing of the numerical attributes of the stimuli, thereby reducing interfering incentive/perceptual effects. The present results are in accordance with this hypothesis. As is illustrated in Figure 1, performance with numeral-candy arrays was more nearly optimal than that with candy-candy pairs [proportion correct for numeralcandy pairs, median $=.70 \pm .045$ interquartile range, as compared with candy-candy arrays, $.19 \pm .025 ; \chi^{2}(d f=1)$ $=5.0, p<.03]$ and was significantly above chance levels $\left[\chi^{2}(d f=1)=10.6, p<.002\right] .^{1}$ Indeed, performance with numeral-candy pairs did not differ significantly from that with numeral-numeral pairs (see Figure 1).

\section{Stimulus Numerosity and Numerical Disparity}

Also similar to our previous results (Boysen et al., 1996), task performance was not uniform across all stimulus pairs but was related to the relative size disparity between the two choice arrays, as well as to overall array sizes. In the previous study, we found that relative performance across array pairs was best organized by a disparity ratio index (numerical disparity between the stimuli/ mean numerical size of the two stimuli). This ratio expresses the numerical disparity between the two stimuli of the pair scaled by the overall numerical size of the stimulus elements. This index incorporates the fact that the effects of a stimulus disparity of 1 on an animal's choice may be quite different for the pair 1-2 than for the pair 4-5. Indeed, in our previous study, the effects of a reward differential were closely described by a Weber-like function $(\Delta$ reward $/$ mean reward $=$ constant $)$. This is consistent 
with the relative, rather than absolute, judgments of the differential incentive values of the choice stimuli, previously reported for both animals and humans (Kreps, 1988; Mitchell, Yao, Sherman, \& O'Regan, 1985; Schrier, 1956).

Figure 2 illustrates task performance as a function of the disparity ratio of the choice stimuli (ranging from .22 for the pair 4-5 to 2.0 for the pair $0-5$ ). As is apparent, performance with candy-candy arrays became progressively worse with higher disparity ratios. The greater the relative reward differential, the more likely it was that the animals would select the larger array and, consequently, receive a smaller reward. The opposite pattern was observed for numeral-numeral pairs. In this case, the larger the reward differential, the more likely the animals were to select the smaller numeral and, thus, receive the larger reward.

Friedman's test revealed significant differences in the slopes for candy-candy and numeral-numeral arrays [for candy-candy, median $=-.13 \pm .16$ interquartile range; for numeral-numeral, median $=+.12 \pm .07 ; \chi^{2}(d f=2)=$ $5.0, p<.03$ ]. The slope function for numeral-candy combinations (median $=-.01 \pm .07$ ) was essentially flat, falling between the candy-candy and the numeralnumeral pairs and not differing significantly from either. ${ }^{2}$

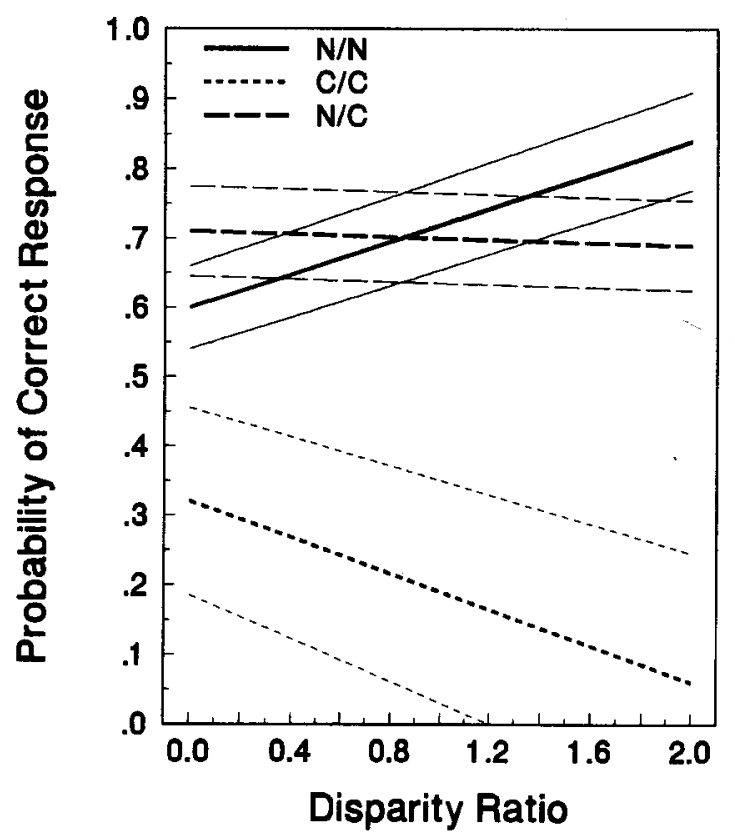

Figure 2. Task performance as a function of the disparity ratio for the paired stimuli. Data illustrate the predominantly negative disparity-ratio slope with candy-candy choice stimuli and the more positive slope with numeral-numeral stimuli. Heavy lines represent the median functions for the numeral-numeral $(N / N)$, number-candy $(\mathrm{N} / \mathrm{C})$, and candy-candy $(\mathrm{C} / \mathrm{C})$ choice stimuli. Corresponding thinner lines depict the interquartile ranges for the individual function of each subject, for the respective condition.

\section{Individual Differences and Sources of Error}

There was remarkable consistency in performance across animals in the candy-candy and numeral-numeral conditions. As was noted, each animal individually was significantly below chance performance when candycandy pairs were used and significantly above chance with numeral-numeral stimuli. More individual differences were apparent with the numeral-candy combinations. The overall group effects on the numeral-candy pairs were carried largely by 3 animals (Kermit, Darrell, and Bobby). The performance of each of these animals was significantly more nearly optimal with numeral-candy pairs than with candy-candy pairs [all $\chi^{2} s(d f=1)>6.0$, $p s<.02]$ and was significantly above the $50 \%$ chance level [ $>70 \%$ correct; all $\left.\chi^{2} \mathrm{~s}(d f=1)>6.4, p s<.02\right]$. The performance of the other 2 animals (Sheba and Sarah) was at chance levels (53\% and $45 \%)$. Additional analyses of error patterns illuminated the bases of these performance differences. Two of the animals displaying performances greater than chance made approximately equal numbers of errors by inappropriately picking the larger number symbol and the larger candy array (average number of errors for selection of the inappropriate numerical stimulus $=7$ trials; selection of the inappropriate candy stimulus $=5$ trials). For these animals, there was no overall bias toward selection of either the candy array or the numerical symbol. The $3 r d$ animal with greater than chance performance did show an apparent bias toward the selection of the candy stimulus (candy was erroneously selected on a total of 11 trials, whereas the numerical stimulus was erroneously selected on only 1 trial). However, the subject was able to overcome this bias on a sufficient number of trials to achieve performance beyond that expected on the basis of chance. Another response strategy would have been to simply select a single stimulus type (which would yield an overall probability correct of .5), an apparent strategy that corresponded with the poor performance of the remaining 2 animals, who performed at chance levels. One of these subjects (Sarah) displayed a strong bias toward selection of the candy stimuli (18 erroneous selections of the candy stimulus vs. 1 erroneous selection of the numeral). The other (Sheba) showed a bias toward selection of the numerals (19 erroneous selections of the numeral vs. 3 erroneous selections of the candy array).

\section{DISCUSSION}

The results of the present study support the proposed hypothesis that the need to process a symbolic stimulus abstractly may reduce interference from associated, but irrelevant, incentive/perceptual features of choice stimuli. In the present study, potential response biases arising from the immediate features of the choice stimuli (i.e., the tendency to select the larger candy array) were pitted against the optimal response strategy (selection of the 
smaller array), on the basis of the experimentally imposed reinforcement contingency. The results are consistent with our earlier studies (Boysen \& Berntson, 1995; Boysen et al., 1996) and suggest that the direct incentive/perceptual features of the choice stimuli introduced a response bias that interfered with a more successful choice strategy based on the differential reward contingency. When the choice stimuli were candy arrays, the animals generally selected the larger array and, thus, consistently received the smaller reward. Performance was significantly below chance, indicating that the subjects were not simply responding randomly. Indeed, the larger the numerical disparity between the candy arrays and, hence, the larger the reward differential, the less near to optimal the choices became. Performance deteriorated under the very conditions in which the animal stood to benefit the most by selecting the smaller array (e.g., one candy vs. six candies).

When the task stimuli were Arabic numerals, however, the chimpanzees were able reliably to select the smaller number and, thus, receive the larger number of candy reinforcers corresponding to the greater numerical value of the remaining symbol. Moreover, in contrast with results with candy-candy choices, performance on numeralnumeral pairs was progressively enhanced with larger disparity ratios and associated reward differentials. This finding suggests that the animals had acquired knowledge of the rule structure of the task but were unable to implement such implicit knowledge because of the competing response biases toward the larger candy arrays.

Worthy of note is the apparent robustness and stability of the task interference effect when candy arrays serve as choice stimuli. All of the present subjects participated in a prior study, completed about 4 years earlier, that included similar candy-candy and numeral-numeral stimulus pairs. Performances were strikingly similar for each stimulus type in the present study. For example, the overall proportion of correct responses with candy-candy arrays was .27 in the prior experiment and .19 in the present study. The results for numeral-numeral stimulus pairs were also comparable (.66 vs. .72), despite the fact that each animal had received over 500 trials on the task. Clearly, the task interference effect with candy arrays is an enduring phenomenon that does not appear to yield to extended learning experience or success with other trial types (numeral-numeral arrays) in the same task with the same reinforcement contingencies.

These findings are reminiscent of the self-control literature in animals and children (Eisenberger, Weier, Masterson, \& Theis, 1989; Logue, 1988; Mischel, Shoda, \& Rodriguez, 1989; Tobin \& Logue, 1994). Young children, for example, have difficulty in inhibiting a direct response to food in order to achieve a larger but delayed reward but respond more optimally when the food stimuli are presented representationally as color slides (Mischel et al., 1989).
Together, these studies suggest that multiple response dispositions may be invoked in behavioral contexts. Often, these dispositions may lead synergistically to concordant behavioral actions and, as Menzel and Draper (1965) suggest, yield commonalities between the direct choice responses of primates and selections based on sign learning. In the present study, however, response biases arising from the apparent incentive/perceptual features of the choice stimuli were directly pitted against the associative disposition based on the reinforcement contingency. Although the animals had apparently acquired the food-distribution rule structure, as evidenced by their immediate and consistently successful responding when numerical stimuli were employed, the direct perceptual/ incentive features of the candy arrays appeared to have introduced a powerful, conflicting disposition. The improved performance with Arabic numbers may be attributable to the fact that these symbolic stimuli failed to evoke such interference. The ability of symbols to encompass selective features or attributes of their realworld referents may constitute an important advantage of symbolic representations. ${ }^{3}$ Symbols may permit an organism to cognitively process selected information and to respond adaptively on the basis of that knowledge structure, while minimizing potential interference from more primitive dispositions. ${ }^{4}$

A primary goal of the present study was to evaluate the hypothesis that inclusion of an abstract numerical symbol as one element of a choice pair could attenuate the interfering response bias associated with a candy array that served as the other choice option. This hypothesis was confirmed. Overall performance with numeralcandy arrays was significantly more optimal than that for candy-candy arrays and did not significantly differ from performance with numeral-numeral pairs. This was a particularly notable finding, because the lack of intrinsic incentive features of the Arabic numerals might be expected to lead to an exaggerated, rather than to a reduced, response bias toward the candy array. It is noteworthy, however, that there were some individual differences in the strategies the animals employed in the numeral-candy condition. These differences are further highlighted by the general similarity in performance measures with candy-candy and numeral-numeral choices. All the animals demonstrated suboptimal performance with candy-candy stimuli but were able to respond more optimally with numeral-numeral stimuli. Each animal was able to effectively process abstract numerical symbols in order to guide task choices to optimize reward. This ability was not sufficient to ensure an optimal selection strategy among numeral-candy choices, however, as the significant group improvement seen over numeral-candy stimuli was carried by 3 of the 5 animals. One of the remaining 2 animals adopted a suboptimal bias toward selecting the candy stimulus regardless of numerosity, whereas the other showed a predominant bias toward the 
numerical stimulus. These biases emerged despite the fact that the animals were able to employ numerical symbols effectively in selecting among numeral- numeral pairs.

The 3 animals that were able to optimally select among the mixed choice stimuli may have had greater capacity for contextual generalization of the abstract enumerative processing of the Arabic symbols. Because numerical symbols are arbitrary, they preclude perceptually based quantity judgment strategies such as subitizing or estimation. Rather, the utility of a symbol depends on its associative link to an abstract representation of numerosity. The requisite abstract processing of the numerical symbols and the task requirement to relate this numerical representation to the paired array stimulus may promote more abstract numerical processing of the candy array stimulus. The basis of such a generalization is unclear, but the symbols may foster an alternative mode of enumeration or quantity judgment of the candy array or may shift attentional focus to the specific dimension of numerosity.

The ability to generalize and benefit from symbolic processing may be enhanced by training on a wide range of cognitive tasks, and some researchers have suggested that language training specifically may promote such capabilities (Premack, 1983a, 1983b, 1984, 1986; SavageRumbaugh, 1986; see Thompson et al., 1997, for a discussion). The latter does not appear to be the critical variable in the present results, however, as the animals who performed the best in the numeral-candy condition had no language training per se. Moreover, the 2 animals with the most suboptimal performance on numeralcandy pairs are among the most cognitively trained chimpanzees in the world (Sheba, from our laboratory, and Sarah, one of the premier language-tutored chimpanzees, who was originally trained in Premack's laboratory prior to transfer to our project in 1987). Age also did not appear to be a primary determinant, as one of the animals displaying the poorest performance was the oldest of the group (Sarah, age 39), and the other was next to the youngest (Sheba, age 16). At present, the functional origins of the animals' performance disparities remain unclear, but differences in attentional processes may warrant further study.

In summary, the incentive and reward determinants of performance may synergistically promote adaptive responding, although in other cases, they may yield striking behavioral conflicts. The nature and manifestations of these conflicts may offer important insights into the cognitive and attentional structure of the animal mind. In view of the potential conflicts between primitive dispositions and those arising from higher cognitive mechanisms, the emergence of the capacity for symbolic representations may reflect an evolutionary strategy for adaptively resolving such conflicts. One potential advantage of symbolic representations is that they may promote abstract cognitive processing of behavioral contexts that may minimize the intrusion of inflexible lower level dis- positions and, thereby, promote optimal responses on the basis of acquired knowledge.

\section{REFERENCES}

Berntson, G. G., Boysen, S. T., \& CACIOPPO, J. T. (1993). Neurobehavioral organization and the cardinal principle of evaluative bivalence. In F. M. Crinella \& J. Yu (Eds.), Brain mechanisms: Papers in memory of Robert Thompson (Annals of the New York Academy of Sciences, Vol. 702, pp. 75-102). New York: New York Academy of Sciences.

BOLLES, R. C. (1970). Species-specific defense reactions and avoidance learning. Psychological Review, 77, 32-48.

Bolles, R. C., \& Fanselow, M. S. (1980). A perceptual-defensiverecuperative model of fear and pain. Behavioral \& Brain Sciences, 3 , 291-301.

Boysen, S. T. (1993). Counting in chimpanzees: Nonhuman principles and emergent properties of number. In S. T. Boysen \& E. J. Capaldi (Eds.), The development of numerical competence: Animal and human models (pp. 39-59). Hillsdale, NJ: Erlbaum.

BOYSEN, S. T. (1997). Representation of quantities by apes. Advances in the Study of Behavior, 26, 435-462.

Boysen, S. T., \& Berntson, G. G. (1989). Numerical competence in a chimpanzee (Pan troglodytes). Journal of Comparative Psychology, 103, 23-31.

Boysen, S. T., \& Berntson, G. G. (1995). Responses to quantity: Perceptual vs. cognitive mechanisms in chimpanzees (Pan troglodytes). Journal of Experimental Psychology: Animal Behavior Processes, 21, 83-86.

Boysen, S. T., Berntson, G. G., Hannan, M. B., \& Cacioppo, J. T. (1996). Quantity-based interference and symbolic representations in chimpanzees (Pan troglodytes). Journal of Experimental Psychology: Animal Behavior Processes, 22, 76-86.

Boysen, S. T., Berntson, G. G., Shreyer, T. A., \& Hannan, M. (1995). Indicating acts during counting by a chimpanzee (Pan troglodytes). Journal of Comparative Psychology, 109, 47-51.

Boysen, S. T., Berntson, G. G., Shreyer, T. A., \& Quigley, K. S. (1993). Processing of ordinality and transitivity by chimpanzees (Pan troglodytes). Journal of Comparative Psychology, 107, 208-215.

Breland, K., \& Breland, M. (1961). The misbehavior of organisms. American Psychologist, 16, 267-269.

CACIOPpo, J. T., \& Berntson, G. G. (1994). Relationship between attitudes and evaluative space: A critical review with emphasis on the separability of positive and negative substrates. Psychological Bulletin, 115, 401-423.

Cacioppo, J. T., Priester, J. R., \& Berntson, G. G. (1993). Rudimentary determinants of attitudes: II. Arm flexion and extension have differential effects on attitudes. Journal of Personality \& Social Psychology: Attitudes \& Social Cognition, 65, 5-17.

Cerutti, D. T., \& Rumbaugh, D. M. (1993). Stimulus relations in comparative primate perspective. Psychological Record, 43, 811-821.

D'amato, M. R., Salmon, D. P., Loukas, E., \& Tomie, A. (1985). Symmetry and transitivity of conditional relations in monkeys $(\mathrm{Ce}-$ bus apella) and pigeons (Columba livia). Journal of the Experimental Analysis of Behavior, 44, 35-47.

Dube, W. V., McIlvane, W. J., Mackay, H. A., \& Stoddard, L. T. (1987). Stimulus class membership established via stimulus-reinforcer relations. Journal of the Experimental Analysis of Behavior, 47, $159-175$.

Eisenberger, R., Weier, F., Masterson, F. A., \& Theis, L. Y. (1989). Fixed-ration schedules increase generalized self-control: Preference for large rewards despite high effort or punishment. Journal of Experimental Psychology: Animal Behavior Processes, 15, 383-392.

FUjiTA, K., \& MATsuzaWA, T. (1986). A new procedure to study the perceptual world of animals with sensory reinforcement: Recognition of humans by a chimpanzee. Primates, 27, 283-291.

Gallistel, C. R. (1993). A conceptual framework for the study of numerical estimation and arithmetic reasoning in animals. In S. T. Boysen \& E. J. Capaldi (Eds.), The development of numerical compe- 
tence: Animal and human models (pp. 211 -223). Hillsdale, NJ: Erlbaum.

Gillan, D. J., Premack, D., \& Woodruff, G. (1981). Reasoning in the chimpanzee: 1. Analogical reasoning. Journal of Experimental Psychology: Animal Behavior Processes, 7, 1-17.

KREPS, D. M. (1988). Notes on the theory of choice. Boulder: Westview.

Limongelli, L., Boysen, S. T., \& Visalberghi, E. (1993). Comprehension of cause-effect relationships in a tool-using task by chimpanzees (Pan troglodytes). Journal of Comparative Psychology, 109, 18-26.

LoGUE, A. W. (1988). Research on self-control: An integrating framework. Behavioral \& Brain Sciences, 11, 665-709.

LoLordo, V. M., \& Droungas, A. (1989). Selective associations and adaptive specializations: Taste aversions and phobias. In S. B. Klein \& R. R. Mowrer (Eds.), Contemporary learning theories: Instrumental conditioning theory and the impact of biological constraints on learning (pp. 145-179). Hillsdale, NJ: Erlbaum.

MatsuZAWA, T. (1985a). Color naming and classification in a chimpanzee (Pan troglodytes). Journal of Human Evolution, 14, 283-291.

MatsuzaWA, T. (1985b). Use of numbers by a chimpanzee. Nature, 315, 57-59.

MCINTiRe, K. D., Cleary, J. P., \& Thompson, T. (1987). Conditional relations by monkeys: Reflexivity, symmetry, and transitivity. Journal of the Experimental Analysis of Behavior, 47, 279-285.

Menzel, E. W., \& Draper, W. A. (1965). Primate selection of food by size: Visible versus invisible rewards. Journal of Comparative \& Physiological Psychology, 59, 231-239.

Mischel, W., ShodA, Y., \& RodRiguez, M. L. (1989). Delay of gratification in children. Science, 244, 933-937.

Mitchell, R. W., Yao, P., Sherman, P. T., \& O'Regan, M. (1985). Discriminative responding of a dolphin (Tursiops truncatus) to differentially rewarded stimuli. Journal of Comparative Psychology, 99 , 218-225.

MUROFUSHI, K. (1997). Numerical matching behavior by a chimpanzee (Pan troglodytes): Subitizing and analogue magnitude estimation. Japanese Psychological Research, 39, 140-153.

PremaCK, D. (1983a). Animal cognition. Annual Review of Psychology, 34, 351-362.

Premack, D. (1983b). The codes of man and beast. Behavioral \& Brain Sciences, 6, 125-137.

Premack, D. (1984). Upgrading a mind. In T. G. Bever, J. M. Carroll, \& L. A. Miller (Eds.), Talking minds: The study of language in cognitive science (pp. 181-206). Cambridge, MA: MIT Press.

Premack, D. (1986). Gavagai. London: Cambridge University Press.

Savage-Rumbaugh, E. S. (1986). Ape language: From conditioned response to symbol. New York: Columbia University Press.

SCHRIER, A. M. (1956). Amount of incentive and performance on a blackwhite discrimination problem. Journal of Comparative \& Physiological Psychology, 43, 123-125.

SEligman, M. E. P. (1970). On the generality of the laws of learning. Psychological Review, 77, 406-418.

Sidman, M., \& TaIlby, W. (1982). Conditional discrimination vs. matching-to-sample: An expansion of the testing paradigm. Journal of the Experimental Analysis of Behavior, 37, 5-22.

THOMPSON, R. K. R., ODEN, D. L., \& BOYSEN, S. T. (1997). Languagenaive chimpanzees (Pan troglodytes) judge relations between rela- tions in a conceptual matching-to-sample task. Journal of Experimental Psychology: Animal Behavior Processes, 23, 31-43.

TIMBERLAKE, W. (1994). Behavior systems, associationism, and Pavlovian conditioning. Psychonomic Bulletin \& Review, 1, 405-420.

Tosin, H., \& Logue, A. W. (1994). Self-control across species (Columba livia, Homo sapiens, and Rattus norvegicus). Journal of Comparative Psychology, 108, 126-133.

\section{NOTES}

1. The poorer performance with candy-candy arrays, relative to numeral-candy pairs, is not likely attributable to a general motivational factor associated with the larger aggregate number of candies in the candy-candy array pairs. A previous study demonstrated that the aggregate size of the arrays was not a reliable predictor of performance (Boysen et al., 1996). Rather, it was the disparity ratio (disparity between the stimuli divided by mean array size) that better accounted for performance. Present results are consistent with this view, as the performance with numeral-candy pairs was not appreciably different when the candy array was large or small. In fact, in numeral-candy trials, performance was slightly better with larger than with smaller candy arrays, although this difference did not achieve significance.

2 . Slopes from the 3 subjects that showed improved performance were not appreciably different from the overall group values for the numeral-candy pairs, but the minimal $N$ of these subgroups precluded meaningful statistical analysis.

3. Consequently, symbols may not be expected to invariably show equivalence relationships with their referents (Cerutti \& Rumbaugh, 1993; D'Amato, Salmon, Loukas, \& Tomie, 1985; McIntire, Cleary, \& Thompson; 1987; Sidman \& Tailby, 1982). Although equivalence relationships can develop between stimuli and associated reinforcers (see, e.g., Dube, McIlvane, Mackay, \& Stoddard, 1987), the present animals had extensive experience and training with Arabic numerals in multiple contexts, and the numerical stimuli were not consistently associated with any specific reinforcer. Rather, the common referent across training contexts was the numerical significance of the Arabic symbols. This is similar to the acquisition and application of number symbols by humans and may account for the apparent lack of incentive interference effects or equivalence relationships with Arabic numerals.

4. Relatively primitive evaluative dispositions may have notable impact on the expression of more complex behaviors related to higher neurobehavioral processes (see, e.g., Timberlake, 1994). In addition to the present data, the concepts of instinctive $\operatorname{drift}(\mathrm{K}$. Breland \& M. Breland, 1961 ), species-specific defense responses (Bolles, 1970; Bolles \& Fanselow, 1980), preparedness and contrapreparedness (Seligman, 1970), and selective associations (LoLordo \& Droungas, 1989) all reflect lower dispositional biases that can shape the likelihood of a specific response or association in a given context. Primitive dispositional biases have also been shown to influence attitudes in humans, as task-irrelevant somatic activity associated with approach or withdrawal reflexes can bias preference judgments toward otherwise neutral stimuli (Cacioppo, Priester, \& Berntson, 1993).

(Manuscript received July 13, 1998; revision accepted for publication November 5, 1998.) 